\title{
Involvement of SAMHD1 in dNTP homeostasis and the maintenance of genomic integrity and oncotherapy (Review)
}

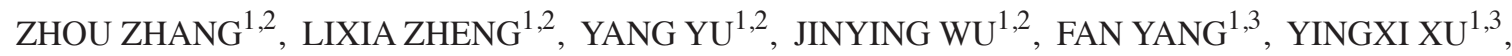

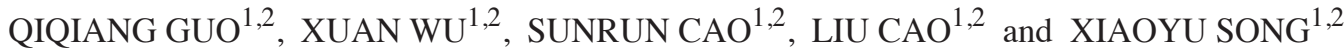 \\ ${ }^{1}$ College of Basic Medical Science, Institute of Translational Medicine, China Medical University, Shenyang, Liaoning 110122; \\ ${ }^{2}$ Liaoning Province Collaborative Innovation Center of Aging Related Disease Diagnosis and Treatment and Prevention, \\ Shenyang, Liaoning $110000 ;{ }^{3}$ Digestive Department, Shengjing Hospital of China Medical University, \\ Shenyang, Liaoning 110122, P.R. China
}

Received October 25, 2019; Accepted February 7, 2020

DOI: $10.3892 / \mathrm{ijo} .2020 .4988$

\begin{abstract}
Sterile alpha motif and histidine/aspartic acid domain-containing protein 1 (SAMHD1), the only deoxynucleotide triphosphate (dNTP) hydrolase in eukaryotes, plays a crucial role in regulating the dynamic balance and ratio of cellular dNTP pools. Furthermore, SAMHD1 has been reported to be involved in the pathological process of several diseases. Homozygous SAMHD1 mutations have been identified in immune system disorders, such as autoimmune disease Aicardi-Goutières syndrome (AGS), whose primary pathogenesis is associated with the abnormal accumulation and disproportion of dNTPs. SAMHD1 is also considered to be an intrinsic virus-restriction factor by suppressing the viral
\end{abstract}

Correspondence to: Dr Xiaoyu Song or Dr Liu Cao, College of Basic Medical Science, Institute of Translational Medicine, China Medical University, 77 Puhe Road, Shenyang, Liaoning 110122, P.R. China

E-mail: xysong@cmu.edu.cn

E-mail: lcao@cmu.edu.cn

Abbreviations: AGS, Aicardi-Goutières syndrome; ARD1, acetyltransferase arrest defective protein 1; BIV, bovine immunodeficiency virus; B-MLV, B-tropic murine leukemia virus; CDK1, cyclin-dependent kinase 1; CLL, chronic lymphocytic leukemia; CTCL, cutaneous T cell lymphoma; CTIP, c-terminal binding protein interacting protein; DDR, DNA damage response; dNTP, deoxynucleotide triphosphate; DSBs, DNA double-strand breaks; EBV, Epstein-Barr virus; EIAV, equine infectious anemia virus; FIV, feline immunodeficiency virus; HBV, hepatitis B virus; HCMV, human cytomegalovirus; HIV, human immunodeficiency virus; HR, homologous recombination; HSV, herpes simplex virus; IFN- $\gamma$, interferon $\gamma$; N-MLV, N-tropic murine leukemia virus; RNase, ribonuclease; SAMHD1, sterile alpha motif and histidine-aspartic acid domain-containing protein 1; SIVs, simian immunodeficiency viruses; Vpr, viral protein $\mathrm{R}$; Vpx, viral protein $\mathrm{X} ;$ 53BP1, p53-binding protein 1

Key words: SAMHD1, dNTP hydrolase, genome integrity, DNA damage response, tumorigenesis infection process, including reverse transcription, replication, packaging and transmission. In addition, SAMHD1 has been shown to promote genome integrity during homologous recombination following DNA damage, thus being considered a promising candidate for oncotherapy applications. The present review summarizes the molecular mechanisms of SAMHD1 regarding the regulation of dNTP homeostasis and DNA damage response. Additionally, its potential effects on tumorigenesis and oncotherapy are reported.

\section{Contents}

1. Introduction

2. Overview of SAMHD1

3. Modifications of SAMHD1

4. Role of SAMHD1 in dNTP homeostasis

5. Role of SAMHD1 in DNA damage response

6. Role of SAMHD1 in immune disorders and viral infections

7. Role of SAMHD1 in tumorigenesis and cancer treatment

8. Conclusions and future perspectives

\section{Introduction}

Deoxynucleotide triphosphates (dNTPs) are the raw materials for DNA replication and repair, rendering them indispensable components for transmitting genetic information in cells and maintaining genomic stability $(1,2)$. Sterile alpha motif and histidine/aspartic acid domain-containing protein 1 (SAMHD1), the only dNTP hydrolase in eukaryotes, is involved in several pathological processes. SAMHD1 is well known for its vital role in the resistance to virus transcription and replication by limiting the volume of the dNTP pool, thus resulting in the protection of the host cellular genome integrity. It has been reported that SAMHD1 acetylation enhances its dNTP hydrolase (dNTPase) activity and regulates cancer cell proliferation (3). Moreobver, the dNTPase activity of SAMHD1 is dependent on the stability of the catalytic core tetramer, which can be inhibited by cyclin-dependent kinase phosphorylation on threonine 592 (T592) (4-8). In addition, viral protein 
kinases can also phosphorylate SAMHD1, thereby inhibiting its dNTPase activity $(9,10)$. The transcriptional repression of Samhdl is mediated by methylation of its promoter (11-13). It has been also reported that viral protein $\mathrm{X}(\mathrm{Vpx})$ interacts with SAMHD1, resulting in the proteasomal degradation of SAMHD1 and an increase in dNTP levels (14-17). Therefore, it is necessary to systematically summarize the modifications of SAMHD1 and reveal its related downstream functions.

In the present review, the current knowledge of the role of SAMHD1 in the dynamic regulation of dNTP cellular homeostasis and genomic stability is summarized. In addition, the potential role of SAMHD1 as a housekeeping protein in the maintenance of dNTP homeostasis and the prevention of tumorigenesis is discussed.

\section{Overview of SAMHD1}

The human SAMHDl gene was first cloned in 2000 by Li et al via a human dendritic cell cDNA library (18) and it was identified as an effective interferon $\gamma($ IFN- $\gamma)$-induced protein $(18,19)$. Based on its dNTPase activity, SAMHD1 is recognized as an intrinsic host restriction factor against human immunodeficiency virus 1 (HIV-1) (20). Additionally, $S A M H D 1$ is also known as the $A G S$ gene. Multipoint mutations in $A G S$ induce severe familial autoimmune Aicardi-Goutières syndrome (AGS) (21-24).

Human SAMHD1 is 626 amino acids (aa) in length and contains an N-terminal nuclear localization domain ${ }^{11} \mathrm{KRPR}^{14}$ followed by a conserved sterile alpha motif (SAM) and a histidine/aspartic acid (HD) domain $(25,26)$. These domains are connected by a short linker and flanked by unstructured regions. The SAM domain (44-110 aa) is involved in protein-protein and protein-DNA/RNA interactions, whereas the HD domain is a conserved sequence containing 160-339 aa comprising an arrangement of alternating histidine/aspartic acid amino acids (27-29) (Fig. 1). HD is the main functional domain of SAMHD1 with antiviral activity, which is involved in nucleotide metabolism and exhibits dNTPase and ribonuclease (RNase) activity. However, the RNase activity of SAMHD1 is controversial. Ryoo et al (31) suggested that SAMHD1 restricted HIV-1 infection by cleaving the viral RNA genome via its RNase activity. In addition, the SAMHD1 phosphorylation at T592 negatively regulates its RNase activity in vivo and impedes HIV-1 restriction. By contrast, Antonucci et al (30) reported that SAMHD1 did not exhibit broad nuclease activity; however, they did not rule out a specific nucleolytic interaction between SAMHD1 and incoming HIV-1 genomic RNA (gRNA). Furthermore, Antonucci et al (30) demonstrated that both SAMHD1 ${ }_{\mathrm{D} 137 \mathrm{~N}}$ (RNase-positive and dNTPase-negative) and $\mathrm{SAMHD} 1_{\mathrm{Q} 548 \mathrm{~A}}$ (RNase-negative and dNTPase-positive) mutants were expressed at comparable levels with wild-type SAMHD1 and each efficiently restricted HIV-1 infection $(30,31)$. Several studies have demonstrated that the C-terminus of SAMHD1 (600-626 aa) is included in the crystal structure of the GTP/dNTP-bound tetramer and forms a short alpha-helical structure with an extended loop (32-34). The C-terminus of SAMHD1 is required for the efficient depletion of dNTP pools and the inhibition of HIV-1 infection in monocytes (35). Although the $\mathrm{C}$-terminal region contains conserved amino acid sequences, it extends interspersed with more divergent ones among vertebrate species (17). A recent study demonstrated that SAMHD1 catalytic activity is regulated by redox signaling. SAMHD1 is inactivated in a dose-dependent, yet reversible manner when treated with the oxidizing agent, $\mathrm{H}_{2} \mathrm{O}_{2}$ (36).

The oxidation of SAMHD1 has been demonstrated to inhibit tetramerization, and has been emphasized as a central regulatory mechanism for the regulation of SAMHD1 activity in vivo (37). Recent research has highlighted that rapid protein degradation is not mediated by SAMHD1 phosphorylation at T592. In addition, it has been documented that the dNTPase activity of SAMHD1 is not only retained during the G1 and G0 phases, but throughout the entire cell cycle, independent of phosphorylation at T592 (38). Other researchers have indicated that constructed mutant SAMHD1 fragments generated by deleting the HD domain and C-terminal segment inhibit the ability to restrict HIV-1 infection (39). In the absence of the dGTP co-factor, SAMHD1 exists as an inactive monomer or dimer in which the substrate-binding pocket is unable to bind dNTP, thus losing its dNTPase activity $(27,35)$. Upon dGTP-Mg ${ }^{2+}$-dGTP binding at the allosteric sites, the catalytically inactive SAMHD1 dimers tetramerize, thereby inducing a large conformational change at the tetramer interface and the recovery of its catalytic activity. Therefore, the dNTPase activity of SAMHD1 is mainly dependent on its active tetramer structure $(35,40)$. Taken together, the aforementioned features of SAMHD1 verify its ability to properly regulate dNTP levels, which are indispensable for the transcription and replication of viruses, such as herpes simplex virus (HSV) type $1(41,42)$ and hepatitis B virus (HBV) $(43,44)$, and the inhibition of HIV-1 reverse transcription. The structure of SAMHD1 forms the basis of its biological functions and may thus provide novel insight into the elucidatation of the internal regulatory mechanisms of immune disorders, viral infections, DNA damage responses and tumorigenesis (45).

\section{Modifications of SAMHD1}

SAMHD1 is subjected to a vast array of post-translational modifications, including phosphorylation, acetylation and methylation. It has been suggested that cyclin-dependent kinase 1 (CDK1)/cyclin A2 phosphorylates SAMHD1 at T592 only in proliferating cells and completely abolish its ability to resist viral infections (46). In addition, SAMHD1 has been shown to be phosphorylated at T592 in proliferating leukocytes in the G1/S and G2/M phase of the cell cycle by the key S-phase kinase complex, CDK2-cyclin A (38). The study by Pauls et al (47) suggested that the CDK6-dependent CDK2 phosphorylation of SAMHD1 inhibited its restriction activity against HIV-1 replication in primary cells. Therefore, the synergistic effect of CDK2 and CDK6 during cell cycle progression is essential for determining the susceptibility to HIV-1 infection by modulating viral dNTP access through SAMHD1. Notably, SAMHD1 is also phosphorylated at T592 from the G0 to G1 phase of the cell cycle through the activation of CDK2 and cyclin E expression, resulting in increased dNTP pools (47).

Furthermore, the levels of SAMHD1 phosphorylation at T592 may be reduced following treatment with type I IFN, reinforcing the link between the phosphorylation of SAMHD1 


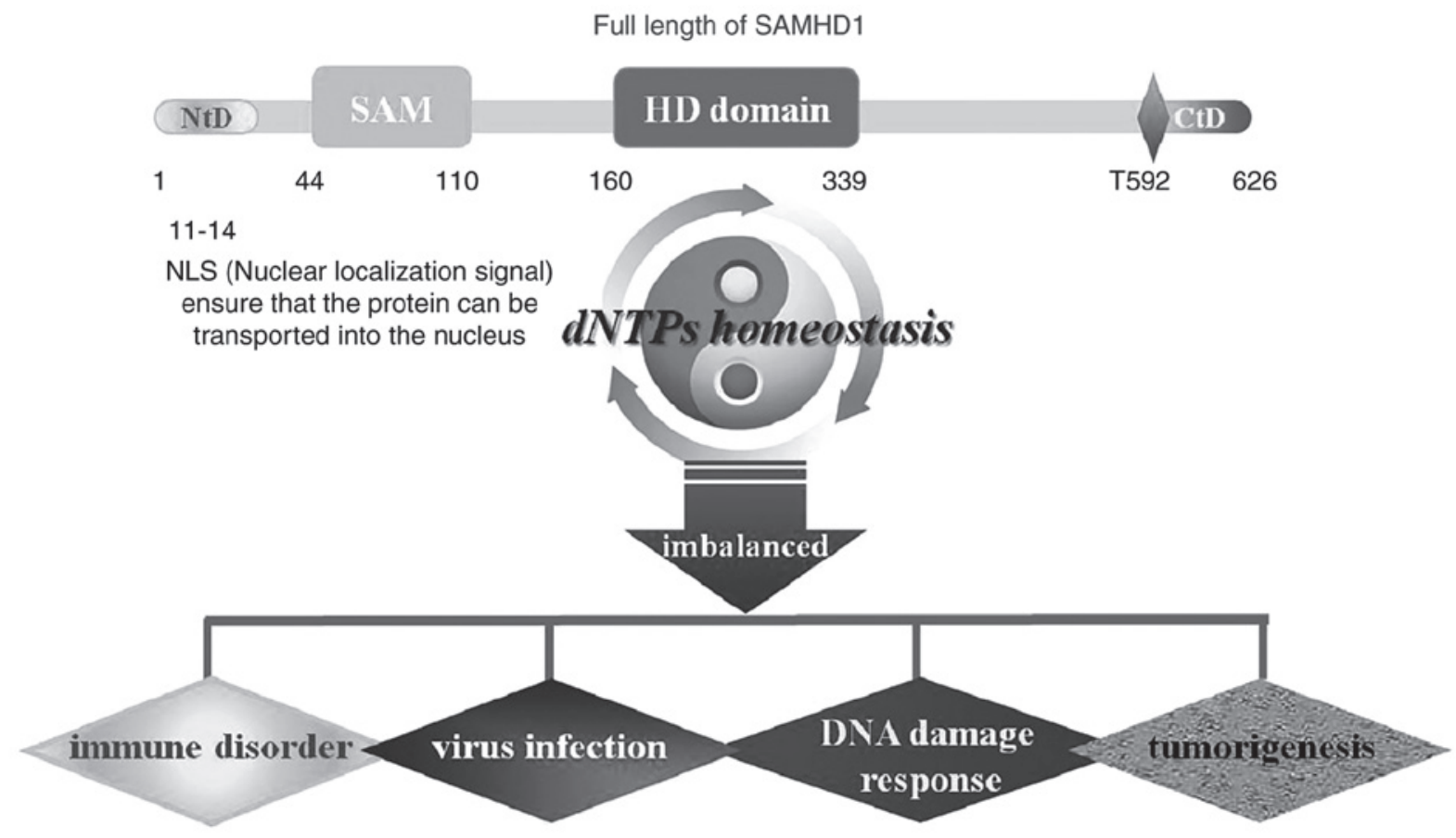

Figure 1. Overview of SAMHD1. SAMHD1, sterile alpha motif and histidine/aspartic acid domain-containing protein 1; dNTPs, deoxynucleotide triphosphates.

and its antiviral activity (46). Recently, several studies have suggested that the expression of $\mathrm{p} 21^{\text {Waf1/Cip1 }}$ (referred to as p21), a CDK inhibitor, may lead to reduced phosphorylation at T592 residue by CDKs. Thus, SAMHD1 antiviral activity is regulated by CDK1 phosphorylation at amino acid T592, and type I IFN renders Vpx unable to induce SAMHD1 degradation (48-50). Type II IFN can stimulate the transcription of SAMHD1 to degrade dNTP and to restrict viral infection positively $(51,52)$ and type III IFN exhibits modest to undetectable activity (53). However, further research is required in this field to explore the underlying molecular biological mechanisms.

The folding of the SAMHD1 region is disrupted around T592E due to negative charge repulsion generated by a phosphomimetic mutation. Subsequently, this disruption leads to the substantial destabilization of the active tetrameric form of SAMHD1 and an approximately 3-fold decrease in its dNTPase activity. However, the T592V variant does not perturb the crystal structure of SAMHD1; thus, the available active SAMHD1 tetramers are not significantly decreased (54). In addition, the importance of SAMHD1 dephosphorylation has also been investigated. Thus, phosphatase PP2A-B55 $\alpha$ is responsible for rendering the antiviral activity of SAMHD1. These results suggest that phosphorylation and dephosphorylation at T592, the key regulatory site of SAMHD1 protein, is responsible for the diverse physiological functions of SAMHD1 (55).

Although alanine substitution at T592 exerts only a minimal effect on the viral restriction ability of SAMHD1 in differentiated U937 cells, phosphomimetic substitution by aspartate and glutamate completely eliminates its antiviral effect. In addition, introducing a T592A alanine mutation does not rescue SAMHD1 restriction in cycling U937 cells, suggesting that the inhibition of phosphorylation is not sufficient to restore SAMHD1 in proliferating cells $(5,39)$. However, the antiviral activity of SAMHD1 is limited to non-cycling cells. As previously mentioned, SAMHD1 is phosphorylated on residue T592 in cycling cells; however, the phosphorylation dissipates when cells are in a non-cycling state, thus modulating the ability of SAMHD1 to block retroviral infection without affecting its dNTPase activity (6).

Moreover, it has been reported that SAMHD1 is acetylated on $\mathrm{K} 405$ by the acetyltransferase arrest defective protein 1 (ARD1) and enhances its dNTPase activity in vitro. However, the non-acetylated arginine substitution mutant (K405R) does not exert a similar effect. Compared with cells expressing wild-type SAMHD1, cancer cells expressing K405R mutant exhibit an attenuated G1/S cell cycle transition and a decreased cell proliferation. SAMHD1 acetylation levels are increased during the G1 phase of the cell cycle. Collectively, these findings suggest that SAMHD1 acetylation enhances its ability to hydrolyze dNTPs and promote cancer cell proliferation. Therefore, SAMHD1 may be a potent effective target for cancer treatment (3).

Finally, it has been documented that promoter hypermethylation suppresses the transcriptional regulation of SAMHD1, thereby downregulating its protein expression and its tumorigenesis-related functions (11-13).

SAMHD1 activity demonstrates a significant association between dNTP homeostasis and disease progression. Thus, further research on the post-translational modifications of SAMHD1 is urgently required in order for its additional benefits to be fully elucidated.

\section{Role of SAMHD1 in dNTP homeostasis}

SAMHD1, as a dNTP hydrolytic enzyme, plays a key role in the maintenance of homeostasis of cellular dNTP pools $(20,56,57)$ and it is essential for preserving genome integrity. It has been 


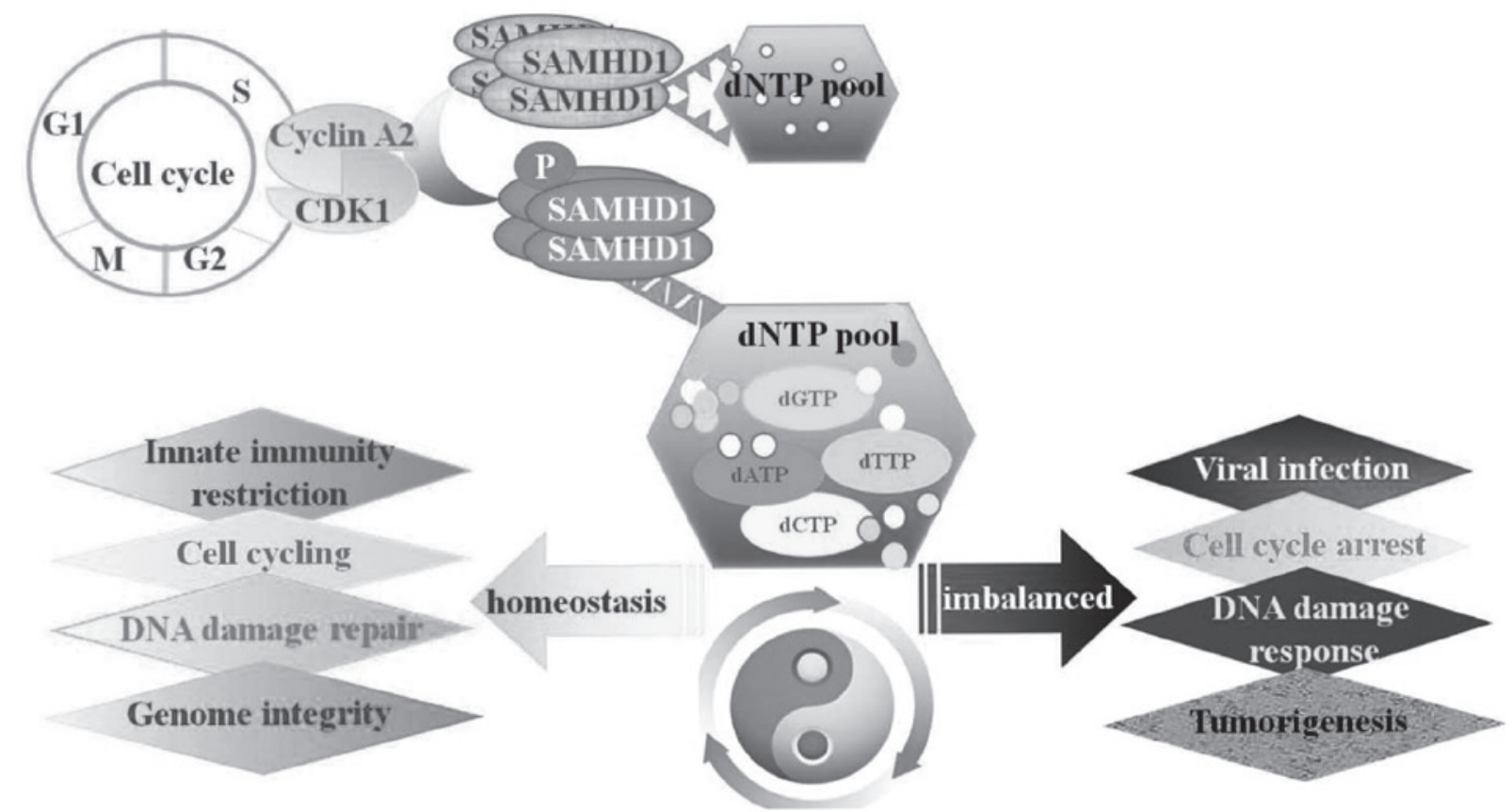

Figure 2. Role of SAMHD1. SAMHD1, sterile alpha motif and histidine/aspartic acid domain-containing protein 1; dNTP, deoxynucleotide triphosphate; CDK1, cyclin-dependent kinase 1 .

reported that dNTP pool imbalance caused by SAMHD1 deficiency may lead to DNA damage, accompanied by the activation of IFN signaling (57). In addition, the surplus of dNTPs induces mismatches and increases the mutation rate during cellular DNA replication (58), which is an important molecular mechanism of tumorigenesis (1). There is increasing evidence to suggest that imbalanced dNTP levels are associated with the rate of replication fork formation under DNA replication stress, leading to gene mutations, genomic instability and cancer development $(59,60)$. Therefore, SAMHD1 is considered a key regulator involved in the maintenance of the dNTP pool and genome homeostasis. The role of SAMHD1 is illustrated in Fig. 2.

\section{Role of SAMHD1 in DNA damage response}

DNA damage in cells, mainly single-strand breaks, arises frequently (approximately 10,000 lesions per cell per day) by a variety of endogenous and exogenous stimuli $(61,62)$. It has been well established that the DNA damage response (DDR) pathway detects lesions in DNA strands and activates the repair system (63). Subsequently, cell cycle checkpoints are activated, providing sufficient time to allow lesions to be repaired. However, an unrepaired or improperly repaired DNA response leads to cell death or abnormal cell mitosis, which may induce malignant transformation and proliferation $(64,65)$. Additionally, inherited defects in DNA damage repair mechanisms are associated with cancer predisposition (66), immunodeficiency (67), neurodegenerative disorders (68), infertility (69) and premature aging, highlighting the critical role of DDR in human health.

Several studies have demonstrated that SAMHD1 participates in the DDR process. Thus, SAMHD1 promotes dNTPase-independent DNA end resection to facilitate
DNA double-strand breaks (DSBs) repair by homologous recombination (HR) (70). In addition, SAMHD1 exhibits a hydrolase-independent function though its C-terminal recruitment of interacting proteins (CTIP) to DSB sites. These observations suggest that SAMHD1 may contribute to anticancer therapy (71). Clifford et al (72) investigated the expression of SAMHD1 in patients with chronic lymphocytic leukemia (CLL) in the UK and revealed that SAMHD1 affected cell proliferation and survival following DNA damage induction. More specifically, the overexpression of wild-type SAMHD1 inhibited proliferation and increased cell death following DSB treatment. Furthermore, SAMHD1 was co-localized with p53-binding protein 1 (53BP1) at the DNA DSB site in the nucleus, which further indicated that SAMHD1 is involved in the DDR process and related diseases (72). By contrast, SAMHD1 downregulation may cause excess dNTPs and a subsequent imbalance of dNTP pools, resulting in base mismatches and mutations during replication, eventually leading to the activation of the intrinsic interferon signal (57). These findings indicate a novel association between SAMHD1 and DDR process in the pathogenesis of several diseases.

\section{Role of SAMHD1 in immune disorders and viral infections}

SAMHD1 is widely expressed in the majority of tissues and cells, and its restrictive function in the innate immunity has been extensively reviewed since it was first discovered. The SAMHD1 gene mutation was detected in autoimmune AGS (22,73-75), which was first described by Jean Aicardi and Francoise Goutières in 1984 (76). The common clinical features of AGS overlap with the autoimmune disease systemic lupus erythematosus (SLE), including brain atrophy and severe sequelae $(75,77,78)$. It has been reported that SAMHD1 
mutations at residues 123, 143, 145, 201, 209, 254, 369 and 385 result in impaired endogenous SAMHD1 protein function and induce nucleotide metabolism disorders in myeloid cells (22). Abnormally increased dNTP pools in fibroblasts derived from patients with AGS are caused by the loss of functional SAMHD1. Subsequently, dNTP accumulation may induce the immune system to secrete excessive amount of antibodies, as it has been previously described (57). These results suggest that SAMHD1 is a key regulator of the immune system by maintaining nucleotide pool homeostasis.

Reverse transcription is a unique DNA synthesis process through which retroviruses and retrotransposons convert single stranded RNA genomes into double stranded DNA. This process is catalyzed by reverse transcriptase, which is a virally encoded DNA polymerase (79,80). Retroviruses consume cellular dNTPs regulated by SAMHD1 to convert their RNA genomes into proviral DNA through reverse transcription (81). DNTPs differ by only a single atom from ribonucleotide triphosphates (NTPs), yet are maintained at 10-1,000-fold lower concentrations (82). Ryoo et al also found that SAMHD1 restricted HIV-1 infection through its RNase activity by cleaving the viral RNA genome, and SAMHD1 associated with HIV-1 RNA and degraded it during the early phases of cell infection (31). The poor dNTP availability in macrophages infected with HIV infection mainly promotes viral mutagenesis induced by frequent rNMP and non-canonical dUMP incorporation $(83,84)$. Finally, SAMHD1 may be a primitive cellular defense tool that was developed to effectively control the replication of dNTP-utilizing pathogens (81).

Human SAMHD1 is a key restriction factor against HIV-1 infection and is highly expressed in non-circulating cells, such as resting $\mathrm{CD}^{+} \mathrm{T}$ cells and terminally differentiated macrophages. SAMHD1 limits HIV-1 infection in non-dividing cells by reducing the levels of intracellular dNTPs during viral reverse transcription, which is indispensable for viral storage and incubation (85). Thus, the overexpression of wild-type SAMHD1 inhibits HIV-1 long terminal repeat (LTR)-driven gene expression at the transcriptional level. In addition, it has been well documented that non-phosphorylated (T592A) and dNTPase inactive [H206D R207N (HD/RN)] mutants of SAMHD1 fail to efficiently inhibit HIV-1 LTR-driven gene expression or the latent virus reactivation (85). SAMHD1 has been reported to be a potent inhibitor of LINE-1 retrotransposition. SAMHD1 is a potent regulator of LINE-1 and LINE-1-mediated Alu/SVA reverse transcriptional transposon. It has also been found that the mutant of SAMHD1 has a defect in LINE-1 inhibition. At the same time, the ability of SAMHD1 to inhibit ORF2p-mediated LINE-1 RNP reverse transcription has been shown to be associated with SAMHD1-mediated LINE-1 inhibition (86). Furthermore, SAMHD1 attenuates IFN- and T-cell-mediated responses by suppressing the induction of virus-specific cytotoxic T-cells in vivo (87). Of note, HIV-2 and simian immunodeficiency viruses (SIVs) with Vpx or viral protein R (Vpr) can induce SAMHD1 degradation, by inhibiting SAMHD1 downregulation during viral infection (25,88-91). Additionally, it has been reported that SAMHD1 blocks feline immunodeficiency virus (FIV), bovine immunodeficiency virus (BIV), equine infectious anemia virus (EIAV), N-tropic murine leukemia virus (N-MLV) and B-tropic murine leukemia virus (B-MLV) infections (92). These findings indicate that SAMHD1 exerts inhibitory effects on infectious diseases.

\section{Role of SAMHD1 in tumorigenesis and cancer treatment}

Recently, SAMHD1 has been shown to be associated with the development of CLL $(72,93)$. Thus, SAMHD1 gene mutations have been detected in leukemia cells, and SAMHD1 mRNA and protein expression levels have been found to be significantly and differentially downregulated. The loss-of-function mutation of SAMHD1 usually occurs early in the evolutionary stage of the molecular cloning of CLL and promotes the development of the disease. In addition, some SAMHD1 mutations are detected in both AGS and CLL, thus AGS patients with SAMHD1 mutations are more likely to suffer concomitant CLL (72).

Several studies have demonstrated that SAMHD1 is associated with the development of multiple types of cancer, such as lung and colon cancer. Thus, in lung adenocarcinoma, SAMHD1 mRNA and protein levels have been shown to be downregulated compared with those noted in adjacent normal tissues. In addition, it has been suggested that the SAMHDI promoter is highly methylated in lung adenocarcinoma, resulting in a suppressed SAMHD1 expression (13). Similarly, frequent mutations in $S A M H D 1$ in colon cancer cells induce SAMHD1 downregulation (94). The aforementioned results suggest that SAMHD1 is closely associated with an increased risk of both lung and colon cancer, and presumably with other types of cancer.

Recently, it was demonstrated that a low level of exogenous SAMHD1 expression can significantly reduce the growth, proliferation and colony formation of HuT78 cells by increasing apoptosis; thus, it may play a potential anticancer role in cutaneous T cell lymphoma (CTCL) (95). In view of the role of SAMHD1 in maintaining genomic stability, it may play an additional role in cells as a cancer suppressor enzyme.

Exogenous SAMHD1 expression in HuT78 cells has also been shown to result in increased spontaneous and Fas ligand (Fas-L)-induced apoptosis levels via the activation of the extrinsic pathway, including caspase-8, -3 and -7 . Mechanistically, SAMHD1 expression in HuT78 cells leads to a significant reduction in the expression of cFLIPS, a key anti-apoptotic regulator that is commonly overexpressed in patients with CTCL (95-97).

The catalogue of somatic mutations in cancer (COSMOS) records 164 unique mutations in SAMHD1 found in samples from various cancer tissues (98). Widely expressed in several tissues, SAMHD1 mutations have also been detected in breast cancer, myeloma, pancreatic cancer and others. The mutation and modification sites of SAMHD1 in different types of cancer are presented in Tables I (99-104) and II, respectively.

The importance of SAMHD1 in dNTP metabolism and genome integrity has been well established; thus, strategies targeting SAMHDI gene replication, post-translational modifications and protein expression have been evaluated for the treatment of cancer and autoimmune diseases (105). SAMHD1 acetylation enhances its dNTPase activity, and thereby, cancer cell arrest at the G1 phase to aid G1/S phase transition and promote cell cycle progression. This observation suggests that the acetylation level of SAMHD1 may be a potential 
Table I. Modifications to SAMHD1 in various human diseases.

\begin{tabular}{|c|c|c|c|}
\hline \multirow[b]{2}{*}{ Type of disease } & \multirow[b]{2}{*}{ Modifications } & Modifications to SAMHD1 & \multirow[b]{2}{*}{ (Refs.) } \\
\hline & & Results & \\
\hline Breast cancer & N.A. & Reduction in protein & $(99)$ \\
\hline Skin T-cell lymphoma & Methylation & Reduction in protein and mRNA & $(95,100)$ \\
\hline Lung cancer & Methylation & Reduction in protein and mRNA & $(101,102)$ \\
\hline Colorectal cancer & N.A. & N.A. & $(94,99,103)$ \\
\hline Cervical cancer & Acetylation & N.A. & (3) \\
\hline HIV-1 & Phosphorylation & Reduction in protein & $(19,53)$ \\
\hline AGS & N.A. & Reduction in protein & $(104)$ \\
\hline $\mathrm{HBV}$ & Phosphorylation & N.A. & $(43,44)$ \\
\hline HSV-1 & Phosphorylation & N.A. & $(41,42)$ \\
\hline EBV & Phosphorylation & N.A. & $(9)$ \\
\hline HCMV & Phosphorylation & N.A. & $(10)$ \\
\hline
\end{tabular}

N.A., no information available; SAMHD1, sterile alpha motif and histidine/aspartic acid domain-containing protein 1; HIV-1, human immunodeficiency virus 1; AGS, Aicardi-Goutières syndrome; HBV, hepatitis B virus; HSV-1, herpes simplex virus 1; EBV, Epstein-Barr virus; HCMV, human cytomegalovirus.

Table II. Mutations of SAMHD1 in different human cancers.

\begin{tabular}{|c|c|c|c|c|c|}
\hline \multirow[b]{2}{*}{ Type of cancers } & \multirow[b]{2}{*}{ Frequency $(\%)$} & \multirow[b]{2}{*}{ Mutation type } & Mutations of $S A M H D 1$ & \multirow[b]{2}{*}{ Results } & \multirow[b]{2}{*}{ (Refs.) } \\
\hline & & & DNA alterations & & \\
\hline Skin cancer & 53.01 & Multipoint & chr20:g.35551400G $>A$ & N.A. & N.A. \\
\hline Liver cancer & 24.42 & Multipoint & chr20:g.35585008A>- & Reduction in protein & $(43)$ \\
\hline Blood cancer & 14.29 & Single point & chr20:g.35559188C>A & N.A. & N.A. \\
\hline Breast cancer & 12.13 & Multipoint & chr20:g.35513711A>- & Reduction in protein & $(99)$ \\
\hline Lung cancer & 11.76 & Multipoint & chr20:g.35518800T>C & Reduction in protein and mRNA & $(13)$ \\
\hline Pancreatic cancer & 7.84 & Multipoint & chr20:g.35519255C >T & N.A. & N.A. \\
\hline Prostate cancer & 1.54 & Single point & chr20:g.35517455T>A & N.A. & N.A. \\
\hline Cervical cancer & 0.52 & Single point & chr20:g.35515883C>G & N.A. & N.A. \\
\hline
\end{tabular}

N.A., no information available; SAMHD1, sterile alpha motif and histidine/aspartic acid domain-containing protein 1.

therapeutic target for cancer treatment. In addition, this finding also unveils a potential method for therapeutically targeting SAMHD1 activity in cells through the use of small molecule inhibitors of acetyltransferases (3). Furthermore, SAMHD1 protects cancer cells from several antinucleoside metabolite treatments, such as cytarabine (Ara-C) which is mainly used in the treatment of acute myeloid leukemia (AML) (106-109). Combination therapy with an anthracycline (commonly doxorubicin or daunorubicin) and Ara-C is the standard treatment for AML (110). Ara-C is converted by the canonical dNTP synthesis pathway to Ara-CTP, the active triphosphate of Ara-C, which serves as a substrate of SAMHD1 (107). Herold et al (106) demonstrated that wild-type SAMHD1 reduced Ara-C treatment efficacy in vivo in an AML mouse model. In addition, THP-1 cells lacking a functional SAMHDI gene have been shown to exhibit an increased sensitivity to antimetabolites, including fludarabine, decitabine, vidarabine and clofarabine (106). SAMHD1 downregulation or the inhibition of its post-translational modifications may be promising strategies with which overcome tumor resistance. Therefore, SAMHD1 is considered a potential biomarker for the stratification of patients with AML and a target for the treatment of Ara-C-refractory AML (109). The aforementioned findings suggest that the invention of a potent SAMHD1 inhibitor that enhances the efficiency of nucleotide analogues should perhaps be a top priority for researchers. Thus, high-throughput assays have already been established from several research groups $(111,112)$. Such approaches seem to be particularly promising for future developments in this field.

\section{Conclusions and future perspectives}

Studies on the unique, natural viral restriction and dNTPase properties of SAMHD1 have demonstrated its involvement in 
the pathogenesis of several diseases and have provided guidance for progress in the development of clinical applications. More specifically, studies on the underlying mechanisms of antiviral agents to fight infection have revealed that SAMHD1 inhibits HIV-1 infection in non-dividing cells by restricting viral reverse transcription, resulting in decreased virus activity and storage $(14,15,113,114)$. In addition, SAMHD1 inhibits SIV activity containing Vpx or $\operatorname{Vpr}(116-118)$.

The dNTPase activity of SAMHD1 maintains balanced cellular dNTP pools, thus preventing genomic instability and tumorigenesis. SAMHD1 loss-of-function mutations are associated with abnormal dNTP accumulation, which induces rapid cancer cell proliferation $(37,105,119,120)$ and immune system disfunctions. On the other hand, SAMHD1 protects cancer cells from DNA replication inhibitors, such as pyrimidine antimetabolite antitumor agents $(104,105)$.

Therefore, future studies on SAMHD1 may provide further insight into the clinical treatment of cancer and other severe diseases. Finally, strategies targeting SAMHD1 are expected to provide more effective health-related interventions.

\section{Acknowledgements}

Not applicable.

\section{Funding}

This study was supported by the National Key R\&D Program of China (2016YFC1302400), the Ministry of Education Innovation Team Development plan to LC(IRT_17R107), and the National Science Foundation of China to XS (31300963, LFWK201725, 2018225083) and QG (81502400).

\section{Availability of data and materials}

Not applicable.

\section{Authors' contributions}

XS and LC designed and conceived the general idea and context of this review article. XS, LC and ZZ conceived and wrote the Abstract. ZZ and YY conceived and wrote the Introduction and 'Overview of SAMHD1' sections. LZ and JW contributed to the 'Modifications of SAMHD1' and the 'Role of SAMHD1 in dNTP homeostasis' sections. FY, YX and QG conducted the writing of the 'Role of SAMHD1 in DNA damage response' and 'Role of SAMHD1 in immune disorders and viral infections' sections. XW and SC completed the 'Role of SAMHD1 in tumorigenesis and cancer treatment' sections. ZZ integrated all sections and relevant references of this manuscript. XS, LC and $\mathrm{ZZ}$ revised the manuscript. All authors read and approved the final manuscript.

\section{Ethics approval and consent to participate}

Not applicable.

\section{Patient consent for publication}

Not applicable.

\section{Competing interests}

The authors declare that they have no competing interests.

\section{References}

1. Kunz BA, Kohalmi SE, Kunkel TA, Mathews CK, McIntosh EM and Reidy JA: International commission for protection against environmental mutagens and carcinogens. Deoxyribonucleoside triphosphate levels: A critical factor in the maintenance of genetic stability. Mutat Res 318: 1-64, 1994.

2. Reichard P: Interactions between deoxyribonucleotide and DNA synthesis. Annu Rev Biochem 57: 349-374, 1988.

3. Lee EJ, Seo JH, Park JH, Vo TTL, An S, Bae SJ, Le H, Lee HS, Wee HJ, Lee D, et al: SAMHD1 acetylation enhances its deoxynucleotide triphosphohydrolase activity and promotes cancer cell proliferation. Oncotarget 8: 68517-68529, 2017.

4. Koharudin LM, Wu Y, DeLucia M, Mehrens J, Gronenborn AM and Ahn J: Structural basis of allosteric activation of sterile $\alpha$ motif and histidine-aspartate domain-containing protein 1 (SAMHD1) by nucleoside triphosphates. J Biol Chem 289: 32617-32627, 2014.

5. Welbourn S, Dutta SM, Semmes OJ and Strebel K: Restriction of virus infection but not catalytic dNTPase activity is regulated by phosphorylation of SAMHD1. J Virol 87: 11516-11524, 2013.

6. White TE, Brandariz-Nunez A, Valle-Casuso JC, Amie S, Nguyen LA, Kim B, Tuzova M and Diaz-Griffero F: The retroviral restriction ability of SAMHD1, but not its deoxynucleotide triphosphohydrolase activity, is regulated by phosphorylation. Cell Host Microbe 13: 441-451, 2013.

7. St Gelais C, de Silva S, Hach JC, White TE, Diaz-Griffero F, Yount JS and Wu L: Identification of cellular proteins interacting with the retroviral restriction factor SAMHD1. J Virol 88: 5834-5844, 2014.

8. Ji X, Tang C, Zhao Q, Wang W and Xiong Y: Structural basis of cellular dNTP regulation by SAMHD1. Proc Natl Acad Sci USA 111: E4305-E4314, 2014.

9. Zhang K, Lv DW and Li R: Conserved herpesvirus protein kinases target SAMHD1 to facilitate virus replication. Cell Rep 28: 449-459 e445, 2019.

10. Kim ET, Roche KL, Kulej K, Spruce LA, Seeholzer SH, Coen DM, Diaz-Griffero F, Murphy EA and Weitzman MD: SAMHD1 modulates early steps during human cytomegalovirus infection by limiting NF-kB activation. Cell Rep 28: 434-448 e436, 2019.

11. de Silva S, Hoy H, Hake TS, Wong HK, Porcu P and Wu L: Promoter methylation regulates SAMHD1 gene expression in human CD4+ T cells. J Biol Chem 288: 9284-9292, 2013.

12. de Silva S, Wang F, Hake TS, Porcu P, Wong HK and Wu L: Downregulation of SAMHD1 expression correlates with promoter DNA methylation in Sezary syndrome patients. J Invest Dermatol 134: 562-565, 2014.

13. Wang JL, Lu FZ, Shen XY, Wu Y and Zhao LT: SAMHD1 is down regulated in lung cancer by methylation and inhibits tumor cell proliferation. Biochem Biophys Res Commun 455: 229-233, 2014.

14. Laguette N, Sobhian B, Casartelli N, Ringeard M, Chable-Bessia C, Ségéral E, Yatim A, Emiliani S, Schwartz O and Benkirane M: SAMHD1 is the dendritic- and myeloid-cell-specific HIV-1 restriction factor counteracted by Vpx. Nature 474: 654-657, 2011

15. Hrecka K, Hao C, Gierszewska M, Swanson SK, Kesik-Brodacka M, Srivastava S, Florens L, Washburn MP and Skowronski J: Vpx relieves inhibition of HIV-1 infection of macrophages mediated by the SAMHD1 protein. Nature 474: 658-661, 2011.

16. Berger A, Sommer AF, Zwarg J, Hamdorf M, Welzel K, Esly N, Panitz S, Reuter A, Ramos I, Jatiani A, et al: SAMHD1-deficient CD14+ cells from individuals with aicardi-goutieres syndrome are highly susceptible to HIV-1 infection. PLoS Pathog 7: e1002425, 2011.

17. Ahn J, Hao C, Yan J, DeLucia M, Mehrens J, Wang C, Gronenborn AM and Skowronski J: HIV/simian immunodeficiency virus (SIV) accessory virulence factor Vpx loads the host cell restriction factor SAMHD1 onto the E3 ubiquitin ligase complex CRL4DCAF1. J Biol Chem 287: 12550-12558, 2012.

18. Li N,Zhang W and Cao X: Identification of human homologue of mouse IFN-gamma induced protein from human dendritic cells. Immunol Lett 74: 221-224, 2000. 
19. Kueck T, Cassella E, Holler J, Kim B and Bieniasz PD: The aryl hydrocarbon receptor and interferon gamma generate antiviral states via transcriptional repression. Elife 7: e38867, 2018.

20. Goldstone DC, Ennis-Adeniran V, Hedden JJ, Groom HC, Rice GI, Christodoulou E, Walker PA, Kelly G, Haire LF, Yap MW, et al: HIV-1 restriction factor SAMHD1 is a deoxynucleoside triphosphate triphosphohydrolase. Nature 480: 379-382, 2011

21. Leshinsky-Silver E, Malinger G, Ben-Sira L, Kidron D, Cohen S, Inbar S, Bezaleli T, Levine A, Vinkler C, Lev D and Lerman-Sagie T: A large homozygous deletion in the SAMHD1 gene causes atypical aicardi-goutieres syndrome associated with mtDNA deletions. Eur J Hum Genet 19: 287-292, 2011.

22. Rice GI, Bond J, Asipu A, Brunette RL, Manfield IW, Carr IM, Fuller JC, Jackson RM, Lamb T, Briggs TA, et al: Mutations involved in aicardi-goutieres syndrome implicate SAMHD1 as regulator of the innate immune response. Nat Genet 41: 829-832, 2009.

23. Thiele H, du Moulin M, Barczyk K, George C, Schwindt W, Nürnberg G, Frosch M, Kurlemann G, Roth J, Nürnberg P and Rutsch F: Cerebral arterial stenoses and stroke: Novel features of Aicardi-Goutieres syndrome caused by the arg164X mutation in SAMHD1 are associated with altered cytokine expression. Hum Mutat 31: E1836-E1850, 2010.

24. Dale RC, Gornall H, Singh-Grewal D, Alcausin M, Rice GI and Crow YJ: Familial aicardi-goutieres syndrome due to SAMHD1 mutations is associated with chronic arthropathy and contractures. Am J Med Genet A 152A: 938-942, 2010.

25. Brandariz-Nunez A, Valle-Casuso JC, White TE, Laguette N, Benkirane M, Brojatsch J and Diaz-Griffero F: Role of SAMHD1 nuclear localization in restriction of HIV-1 and SIVmac. Retrovirology 9: 49, 2012

26. Hofmann H, Logue EC, Bloch N, Daddacha W, Polsky SB, Schultz ML, Kim B and Landau NR: The Vpx lentiviral accessory protein targets SAMHD1 for degradation in the nucleus. J Virol 86: 12552-12560, 2012.

27. DeLucia M, Mehrens J, Wu Y and Ahn J: HIV-2 and SIVmac accessory virulence factor Vpx down-regulates SAMHD1 enzyme catalysis prior to proteasome-dependent degradation J Biol Chem 288: 19116-19126, 2013.

28. Kim CA and Bowie JU: SAM domains: Uniform structure, diversity of function. Trends Biochem Sci 28: 625-628, 2003

29. Laguette $\mathrm{N}$ and Benkirane M: How SAMHD1 changes our view of viral restriction. Trends Immunol 33: 26-33, 2012.

30. Antonucci JM, St Gelais C, de Silva S, Yount JS, Tang C, Ji X, Shepard C, Xiong Y, Kim B and Wu L: SAMHD1-mediated HIV-1 restriction in cells does not involve ribonuclease activity. Nat Med 22: 1072-1074, 2016.

31. Ryoo J, Choi J, Oh C, Kim S, Seo M, Kim SY, Seo D, Kim J, White TE, Brandariz-Nuñez A, et al: The ribonuclease activity of SAMHD1 is required for HIV-1 restriction. Nat Med 20: 936-941, 2014.

32. Zhu CF, Wei W, Peng X, Dong YH, Gong Y and Yu XF The mechanism of substrate-controlled allosteric regulation of SAMHD1 activated by GTP. Acta Crystallogr D Biol Crystallogr 71: 516-524, 2015.

33. Li Y, Kong J, Peng X, Hou W, Qin X and Yu XF: Structural insights into the high-efficiency catalytic mechanism of the sterile $\alpha$-motif/histidine-aspartate domain-containing protein. J Biol Chem 290: 29428-29437, 2015.

34. Patra KK, Bhattacharya A and Bhattacharya S: Allosteric signal transduction in HIV-1 restriction factor SAMHD1 proceeds via reciprocal handshake across monomers. J Chem Inf Model 57: 2523-2538, 2017

35. Yan J, Kaur S, DeLucia M,Hao C, Mehrens J, Wang C, Golczak M, Palczewski K, Gronenborn AM, Ahn J and Skowronski J: Tetramerization of SAMHD1 is required for biological activity and inhibition of HIV infection. J Biol Chem 288: 10406-10417, 2013.

36. Mauney CH, Rogers LC,Harris RS, Daniel LW, Devarie-Baez NO, Wu H, Furdui CM, Poole LB, Perrino FW and Hollis T: The SAMHD1 dNTP triphosphohydrolase is controlled by a redox switch. Antioxid Redox Signal 27: 1317-1331, 2017.

37. Mauney $\mathrm{CH}$ and Hollis T: SAMHD1: Recurring roles in cell cycle, viral restriction, cancer, and innate immunity. Autoimmunity 51: 96-110, 2018

38. Tramentozzi E, Ferraro P, Hossain M, Stillman B, Bianchi V and Pontarin G: The dNTP triphosphohydrolase activity of SAMHD1 persists during S-phase when the enzyme is phosphorylated at T592. Cell Cycle 17: 1102-1114, 2018
39. Arnold LH, Groom HC, Kunzelmann S, Schwefel D, Caswell SJ, Ordonez P, Mann MC, Rueschenbaum S, Goldstone DC, Pennell S, et al: Phospho-dependent regulation of SAMHD1 oligomerisation couples catalysis and restriction. PLoS Pathog 11: e1005194, 2015.

40. Ji X, Wu Y, Yan J, Mehrens J, Yang H, DeLucia M, Hao C, Gronenborn AM, Skowronski J, Ahn J and Xiong Y: Mechanism of allosteric activation of SAMHD1 by dGTP. Nat Struct Mol Biol 20: 1304-1309, 2013

41. Badia R, Angulo G, Riveira-Munoz E, Pujantell M, Puig T, Ramirez C, Torres-Torronteras J, Martí R, Pauls E, Clotet B, et al: Inhibition of herpes simplex virus type 1 by the CDK6 inhibitor PD-0332991 (palbociclib) through the control of SAMHD1. J Antimicrob Chemother 71: 387-394, 2016.

42. Kim ET, White TE, Brandariz-Nunez A, Diaz-Griffero F and Weitzman MD: SAMHD1 restricts herpes simplex virus 1 in macrophages by limiting DNA replication. J Virol 87: 12949-12956, 2013.

43. Hu J, Qiao M, Chen Y, Tang H, Zhang W, Tang D, Pi S, Dai J, Tang N, Huang A and Hu Y: Cyclin E2-CDK2 mediates SAMHD1 phosphorylation to abrogate its restriction of HBV replication in hepatoma cells. FEBS Lett 592: 1893-1904, 2018.

44. Sommer AF, Riviere L, Qu B, Schott K, Riess M, Ni Y, Shepard C, Schnellbächer E, Finkernagel M, Himmelsbach K, et al: Restrictive influence of SAMHD1 on Hepatitis B Virus life cycle. Sci Rep 6: 26616, 2016.

45. Li M, Zhang D, Zhu M, Shen Y, Wei W, Ying S, Korner H and Li J: Roles of SAMHD1 in antiviral defense, autoimmunity and cancer. Rev Med Virol 27: 2017.

46. Cribier A, Descours B, Valadao AL, Laguette N and Benkirane M: Phosphorylation of SAMHD1 by Cyclin A2/CDK1 regulates its restriction activity toward HIV-1. Cell Rep 3: 1036-1043, 2013.

47. Pauls E, Ruiz A, Badia R, Permanyer M, Gubern A, Riveira-Muñoz E, Torres-Torronteras J, Alvarez M, Mothe B, Brander C, et al: Cell cycle control and HIV-1 susceptibility are linked by CDK6-dependent CDK2 phosphorylation of SAMHD1 in myeloid and lymphoid cells. J Immunol 193: 1988-1997, 2014.

48. Valle-Casuso JC, Allouch A, David A, Lenzi GM, Studdard L, Barré-Sinoussi F, Müller-Trutwin M, Kim B, Pancino G and Sáez-Cirión A: p21 Restricts HIV-1 in monocyte-derived dendritic cells through the reduction of deoxynucleoside triphosphate biosynthesis and regulation of SAMHD1 antiviral activity. J Virol 91: e01324-e01317, 2017.

49. Bloch N, O'Brien M, Norton TD, Polsky SB, Bhardwaj N and Landau NR: HIV type 1 infection of plasmacytoid and myeloid dendritic cells is restricted by high levels of SAMHD1 and cannot be counteracted by Vpx. AIDS Res Hum Retroviruses 30: 195-203, 2014.

50. Dragin L, Nguyen LA, Lahouassa H, Sourisce A, Kim B, Ramirez BC and Margottin-Goguet F: Interferon block to HIV-1 transduction in macrophages despite SAMHD1 degradation and high deoxynucleoside triphosphates supply. Retrovirology 10: 30, 2013.

51. Lafuse WP, Brown D, Castle L and Zwilling BS: Cloning and characterization of a novel cDNA that is IFN-gamma-induced in mouse peritoneal macrophages and encodes a putative GTP-binding protein. J Leukoc Biol 57: 477-483, 1995.

52. Taylor GA, Jeffers M, Largaespada DA, Jenkins NA, Copeland NG and Vande Woude GF: Identification of a novel GTPase, the inducibly expressed GTPase, that accumulates in response to interferon gamma. J Biol Chem 271: 20399-20405, 1996.

53. Szaniawski MA, Spivak AM, Cox JE, Catrow JL, Hanley T, Williams ESCP, Tremblay MJ, Bosque A and Planelles V: SAMHD1 phosphorylation coordinates the Anti-HIV-1 response by diverse interferons and tyrosine kinase inhibition. Mbio 9 : e00819-e00818, 2018.

54. Tang C, Ji X, Wu L and Xiong Y: Impaired dNTPase activity of SAMHD1 by phosphomimetic mutation of Thr-592. J Biol Chem 290: 26352-26359, 2015

55. Schott K, Fuchs NV, Derua R, Mahboubi B, Schnellbächer E, Seifried J, Tondera C, Schmitz H, Shepard C, Brandariz-Nuñez A, et al: Dephosphorylation of the HIV-1 restriction factor SAMHD1 is mediated by PP2A-B55 $\alpha$ holoenzymes during mitotic exit. Nat Commun 9: 2227, 2018.

56. Franzolin E, Pontarin G, Rampazzo C, Miazzi C, Ferraro P, Palumbo E, Reichard P and Bianchi V: The deoxynucleotide triphosphohydrolase SAMHD1 is a major regulator of DNA precursor pools in mammalian cells. Proc Natl Acad Sci USA 110: 14272-14277, 2013 
57. Kretschmer S, Wolf C, Konig N, Staroske W, Guck J, Häusler M, Luksch H, Nguyen LA, Kim B, Alexopoulou D, et al: SAMHD1 prevents autoimmunity by maintaining genome stability. Ann Rheum Dis 74: e17, 2015.

58. Mathews CK: DNA precursor metabolism and genomic stability. FASEB J 20: 1300-1314, 2006.

59. Poli J, Tsaponina O, Crabbe L, Keszthelyi A, Pantesco V, Chabes A, Lengronne A and Pasero P: dNTP pools determine fork progression and origin usage under replication stress. EMBO J 31: 883-894, 2012.

60. Coquel F, Silva MJ, Techer H, Zadorozhny K, Sharma S, Nieminuszczy J, Mettling C, Dardillac E, Barthe A, Schmitz AL, et al: SAMHD1 acts at stalled replication forks to prevent interferon induction. Nature 557: 57-61, 2018.

61. Seo YR, Sweeney C and Smith ML: Selenomethionine induction of DNA repair response in human fibroblasts. Oncogene 21: 3663-3669, 2002.

62. Lin Y,Ha A and Yan S: Methods for studying DNA single-strand break repair and signaling in xenopus laevis egg extracts. Methods Mol Biol 1999: 161-172, 2019.

63. Hanawalt PC: Historical perspective on the DNA damage response. DNA Repair (Amst) 36: 2-7, 2015.

64. Chu G: Double strand break repair. J Biol Chem 272: 24097-24100, 1997

65. Rooney S, Chaudhuri J and Alt FW: The role of the non-homologous end-joining pathway in lymphocyte development. Immunol Rev 200: 115-131, 2004.

66. Figueroa-Gonzalez G and Perez-Plasencia C: Strategies for the evaluation of DNA damage and repair mechanisms in cancer. Oncol Lett 13: 3982-3988, 2017

67. Morio T: Recent advances in the study of immunodeficiency and DNA damage response. Int J Hematol 106: 357-365, 2017.

68. Barzilai A: DNA damage, neuronal and glial cell death and neurodegeneration. Apoptosis 15: 1371-1381, 2010.

69. Brown JS and Jackson SP: Ubiquitylation, neddylation and the DNA damage response. Open Biol 5: 150018, 2015.

70. Medeiros AC, Soares CS, Coelho PO, Vieira NA, Baqui MMA, Teixeira FR and Gomes MD: DNA damage response signaling does not trigger redistribution of SAMHD1 to nuclear foci. Biochem Biophys Res Commun 499: 790-796, 2018.

71. Cabello-Lobato MJ, Wang S and Schmidt CK: SAMHD1 sheds moonlight on DNA double-strand break repair. Trends Genet 33: 895-897, 2017.

72. Clifford R, Louis T, Robbe P, Ackroyd S, Burns A, Timbs AT, Wright Colopy G, Dreau H, Sigaux F, Judde JG, et al: SAMHD1 is mutated recurrently in chronic lymphocytic leukemia and is involved in response to DNA damage. Blood 123: 1021-1031, 2014.

73. Oh C, Ryoo J, Park K, Kim B, Daly MB, Cho D and Ahn K A central role for PI3K-AKT signaling pathway in linking SAMHD1-deficiency to the type I interferon signature. Sci Rep 8: 84, 2018.

74. Martinez-Lopez A, Martin-Fernandez M, Buta S, Kim B, Bogunovic D and Diaz-Griffero F: SAMHD1 deficient human monocytes autonomously trigger type I interferon. Mol Immunol 101: 450-460, 2018.

75. Ramantani G, Kohlhase J, Hertzberg C, Innes AM, Engel K, Hunger S, Borozdin W, Mah JK, Ungerath K, Walkenhorst $\mathrm{H}$, et al: Expanding the phenotypic spectrum of lupus erythematosus in aicardi-goutieres syndrome. Arthritis Rheum 62: 1469-1477, 2010

76. Aicardi J and Goutieres F: A progressive familial encephalopathy in infancy with calcifications of the basal ganglia and chronic cerebrospinal fluid lymphocytosis. Ann Neurol 15: 49-54, 1984.

77. Pendergraft WF III and Means TK: AGS, SLE, and RNASEH2 mutations: Translating insights into therapeutic advances. J Clin Invest 125: 102-104, 2015

78. Ramantani G, Hausler M, Niggemann P, Wessling B Guttmann H, Mull M, Tenbrock K and Lee-Kirsch MA: Aicardi-Goutieres syndrome and systemic lupus erythematosus (SLE) in a 12-year-old boy with SAMHD1 mutations. J Child Neurol 26: 1425-1428, 2011.

79. Hu WS and Hughes SH: HIV-1 reverse transcription. Cold Spring Harb Perspect Med 2: a006882, 2012.

80. Sarafianos SG, Marchand B, Das K, Himmel DM, Parniak MA, Hughes SH and Arnold E: Structure and function of HIV-1 reverse transcriptase: Molecular mechanisms of polymerization and inhibition. J Mol Biol 385: 693-713, 2009.

81. Amie SM, Noble E and Kim B: Intracellular nucleotide levels and the control of retroviral infections. Virology 436: 247-254, 2013.
82. Traut TW: Physiological concentrations of purines and pyrimidines. Mol Cell Biochem 140: 1-22, 1994.

83. Kennedy EM, Amie SM, Bambara RA and Kim B: Frequent incorporation of ribonucleotides during HIV-1 reverse transcription and their attenuated repair in macrophages. J Biol Chem 287: 14280-14288, 2012

84. Kennedy EM, Gavegnano C, Nguyen L, Slater R, Lucas A, Fromentin E, Schinazi RF and Kim B: Ribonucleoside triphosphates as substrate of human immunodeficiency virus type 1 reverse transcriptase in human macrophages. J Biol Chem 285: 39380-39391, 2010.

85. Antonucci JM, Kim SH, St Gelais C, Bonifati S, Li TW, Buzovetsky O, Knecht KM, Duchon AA, Xiong Y, Musier-Forsyth K and Wu L: SAMHD1 impairs HIV-1 gene expression and negatively modulates reactivation of viral latency in CD4(+) T cells. J Virol 92: e00292-e00218, 2018.

86. Gao W, Li G, Bian X, Rui Y, Zhai C, Liu P, Su J, Wang H, Zhu C, Du Y, et al: Defective modulation of LINE-1 retrotransposition by cancer-associated SAMHD1 mutants. Biochem Biophys Res Commun 519: 213-219, 2019.

87. Maelfait J,Bridgeman A, Benlahrech A, Cursi C and Rehwinkel J: Restriction by SAMHD1 limits cGAS/STING-dependent innate and adaptive immune responses to HIV-1. Cell Rep 16: $1492-1501,2016$

88. Baldauf HM, Stegmann L, Schwarz SM, Ambiel I, Trotard M, Martin M, Burggraf M, Lenzi GM, Lejk H, Pan X, et al: Vpx overcomes a SAMHD1-independent block to HIV reverse transcription that is specific to resting CD4 T cells. Proc Natl Acad Sci USA 114: 2729-2734, 2017

89. Miyakawa K, Matsunaga S, Yokoyama M, Nomaguchi M, Kimura Y, Nishi M, Kimura H, Sato H, Hirano H, Tamura T, et al: PIM kinases facilitate lentiviral evasion from SAMHD1 restriction via Vpx phosphorylation. Nat Commun 10: 1844, 2019.

90. Yurkovetskiy L, Guney MH, Kim K, Goh SL, McCauley S, Dauphin A, Diehl WE and Luban J: Primate immunodeficiency virus proteins Vpx and Vpr counteract transcriptional repression of proviruses by the HUSH complex. Nat Microbiol 3: 1354-1361, 2018.

91. Reinhard C, Bottinelli D, Kim B and Luban J: Vpx rescue of HIV-1 from the antiviral state in mature dendritic cells is independent of the intracellular deoxynucleotide concentration. Retrovirology 11: 12, 2014.

92. White TE, Brandariz-Nunez A, Valle-Casuso JC, Amie S, Nguyen L, Kim B, Brojatsch J and Diaz-Griffero F: Contribution of SAM and HD domains to retroviral restriction mediated by human SAMHD1. Virology 436: 81-90, 2013.

93. Rossi D: SAMHD1: A new gene for CLL. Blood 123: 951-952, 2014

94. Rentoft M, Lindell K, Tran P, Chabes AL, Buckland RJ, Watt DL, Marjavaara L, Nilsson AK, Melin B, Trygg J, et al: Heterozygous colon cancer-associated mutations of SAMHD1 have functional significance. Proc Natl Acad Sci USA 113: 4723-4728, 2016.

95. Kodigepalli KM, Li MH, Liu SL and Wu L: Exogenous expression of SAMHD1 inhibits proliferation and induces apoptosis in cutaneous T-cell lymphoma-derived HuT78 cells. Cell Cycle 16: 179-188, 2017.

96. Contassot E, Kerl K, Roques S, Shane R, Gaide O, Dupuis M, Rook AH and French LE: Resistance to FasL and tumor necrosis factor-related apoptosis-inducing ligand-mediated apoptosis in Sezary syndrome T-cells associated with impaired death receptor and FLICE-inhibitory protein expression. Blood 111: 4780-4787, 2008

97. Zhang CL, Kamarashev J, Qin JZ, Burg G, Dummer R and Dobbeling U: Expression of apoptosis regulators in cutaneous T-cell lymphoma (CTCL) cells. J Pathol 200: 249-254, 2003.

98. Forbes SA, Beare D, Boutselakis H, Bamford S, Bindal N, Tate J, Cole CG, Ward S, Dawson E, Ponting L, et al: COSMIC: Somatic cancer genetics at high-resolution. Nucleic Acids Res 45: D777-D783, 2017.

99. Sjoblom T, Jones S, Wood LD, Parsons DW, Lin J, Barber TD, Mandelker D, Leary RJ, Ptak J, Silliman N, et al: The consensus coding sequences of human breast and colorectal cancers. Science 314: 268-274, 2006.

100. Kohnken R, Kodigepalli KM, Mishra A, Porcu P and Wu L: MicroRNA-181 contributes to downregulation of SAMHD1 expression in CD4+T-cells derived from Sezary syndrome patients. Leuk Res 52: 58-66, 2017.

101. Liu J, Lee W, Jiang Z, Chen Z, Jhunjhunwala S, Haverty PM, Gnad F, Guan Y, Gilbert HN, Stinson J, et al: Genome and transcriptome sequencing of lung cancers reveal diverse mutational and splicing events. Genome Res 22: 2315-2327, 2012. 
102. Shang Z, Qian L, Liu S, Niu X, Qiao Z, Sun Y, Zhang Y, Fan LY, Guan X, Cao CX and Xiao H: Graphene oxide-facilitated comprehensive analysis of cellular nucleic acid binding proteins for lung cancer. Acs Appl Mater Interfaces 10: 17756-17770, 2018.

103. Yang CA, Huang HY, Chang YS, Lin CL, Lai IL and Chang JG: DNA-sensing and nuclease gene expressions as markers for colorectal cancer progression. Oncology 92: 115-124, 2017.

104. Herrmann A, Wittmann S, Thomas D, Shepard CN, Kim B, Ferreirós $\mathrm{N}$ and Gramberg T: The SAMHD1-mediated block of LINE-1 retroelements is regulated by phosphorylation. Mob DNA 9: 11, 2018.

105. Kohnken R, Kodigepalli KM and Wu L: Regulation of deoxynucleotide metabolism in cancer: Novel mechanisms and therapeutic implications. Mol Cancer 14: 176, 2015.

106. Herold N, Rudd SG, Sanjiv K, Kutzner J, Bladh J, Paulin CBJ, Helleday T, Henter JI and Schaller T: SAMHD1 protects cancer cells from various nucleoside-based antimetabolites. Cell Cycle 16: 1029-1038, 2017

107. Rudd SG, Schaller T and Herold N: SAMHD1 is a barrier to antimetabolite-based cancer therapies. Mol Cell Oncol 4: e1287554, 2017.

108. Zhu KW, Chen P, Zhang DY, Yan H, Liu H, Cen LN, Liu YL, Cao S, Zhou G, Zeng H, et al: Association of genetic polymorphisms in genes involved in Ara-C and dNTP metabolism pathway with chemosensitivity and prognosis of adult acute myeloid leukemia (AML). J Transl Med 16: 90, 2018.

109. Schneider C, Oellerich T, Baldauf HM, Schwarz SM, Thomas D, Flick R, Bohnenberger H, Kaderali L, Stegmann L, Cremer A, et al: SAMHD1 is a biomarker for cytarabine response and a therapeutic target in acute myeloid leukemia. Nat Med 23: 250-255, 2017.

110. Ossenkoppele $\mathrm{G}$ and Lowenberg B: How I treat the older patient with acute myeloid leukemia. Blood 125: 767-774, 2015.

111. Arnold LH, Kunzelmann S, Webb MR and Taylor IA: A continuous enzyme-coupled assay for triphosphohydrolase activity of HIV-1 restriction factor SAMHD1. Antimicrob Agents Chemother 59: 186-192, 2015
112. Seamon KJ and Stivers JT: A high-throughput enzyme-coupled assay for SAMHD1 dNTPase. J Biomol Screen 20: 801-809, 2015.

113. Baldauf HM, Pan X, Erikson E, Schmidt S, Daddacha W, Burggraf $M$, Schenkova K, Ambiel I, Wabnitz G, Gramberg T, et al: SAMHD1 restricts HIV-1 infection in resting CD4(+) T cells. Nat Med 18: 1682-1687, 2012.

114. Descours B, Cribier A, Chable-Bessia C, Ayinde D, Rice G, Crow Y, Yatim A, Schwartz O, Laguette N and Benkirane M: SAMHD1 restricts HIV-1 reverse transcription in quiescent CD4(+) T-cells. Retrovirology 9: 87, 2012.

115. Lahouassa H, Daddacha W, Hofmann H, Ayinde D, Logue EC, Dragin L, Bloch N, Maudet C, Bertrand M, Gramberg T, et al: SAMHD1 restricts the replication of human immunodeficiency virus type 1 by depleting the intracellular pool of deoxynucleoside triphosphates. Nat Immunol 13: 223-228, 2012.

116. Sakai Y, Doi N, Miyazaki Y, Adachi A and Nomaguchi M: Phylogenetic insights into the functional relationship between primate lentiviral reverse transcriptase and accessory proteins vpx/vpr. Front Microbiol 7: 1655, 2016.

117. Plitnik T, Sharkey ME, Mahboubi B, Kim B and Stevenson M: Incomplete suppression of hiv-1 by samhd1 permits efficient macrophage infection. Pathog Immun 3: 197-223, 2018.

118. Mereby SA, Maehigashi T, Holler JM, Kim DH, Schinazi RF and Kim B: Interplay of ancestral non-primate lentiviruses with the virus-restricting SAMHD1 proteins of their hosts. J Biol Chem 293: 16402-16412, 2018.

119. Wang Z, Bhattacharya A, Villacorta J, Diaz-Griffero F and Ivanov DN: Allosteric activation of SAMHD1 protein by deoxynucleotide triphosphate (dNTP)-dependent tetramerization requires dNTP concentrations that are similar to dNTP concentrations observed in cycling T cells. J Biol Chem 291: 21407-21413, 2016.

120. Bonifati S, Daly MB, St Gelais C, Kim SH, Hollenbaugh JA, Shepard C, Kennedy EM, Kim DH, Schinazi RF, Kim B and Wu L: SAMHD1 controls cell cycle status, apoptosis and HIV-1 infection in monocytic THP-1 cells. Virology 495: 92-100, 2016. 